\title{
MRI for the evaluation of pectus excavatum
}

\author{
Peter A. Marcovici • Barry E. LoSasso • Peter Kruk • \\ Jerry R. Dwek
}

Received: 4 November 2010 /Revised: 30 December 2010 / Accepted: 5 January 2011 /Published online: 8 March 2011

(C) The Author(s) 2011. This article is published with open access at Springerlink.com

\begin{abstract}
Pectus excavatum, the most common congenital deformity of the anterior chest wall, is both a cosmetic and functional abnormality. The degree of abnormal chest wall deformity determines its functional effect, particularly its cardiac and pulmonary impact. Although CT scanning is the most widely used cross-sectional imaging technique used to measure the Haller index, the radiation exposure is reason to seek other alternatives. At our institution, we have introduced a rapid MRI technique for this purpose, which utilizes a single-axial 2-D FIESTA acquisition.
\end{abstract}

Keywords Pectus excavatum $\cdot$ MRI $\cdot$ Haller index

\section{Introduction}

Pectus excavatum, the most common congenital deformity of the anterior chest wall, is both a cosmetic and functional abnormality. The degree of abnormal chest wall deformity determines its functional effect, particularly its cardiac and pulmonary impact.

The diagnosis of pectus excavatum is clinical. However, the quantitative measurement of the deformity is measured using cross-sectional imaging. In particular, the commonly

P. A. Marcovici $(\bowtie)$

Department of Radiology, University of California, San Diego, 200 W. Arbor Dr.,

San Diego, CA 92103-8756, USA

e-mail: pmarcovici@ucsd.edu

B. E. LoSasso

Department of Surgery, Children's Hospital and Health Center, San Diego, CA, USA

P. Kruk · J. R. Dwek

Department of Radiology, Children's Hospital and Health Center, San Diego, CA, USA used Haller index is the ratio between the lateral distance of the chest wall (inner margins) and the narrowest anteroposterior distance between the vertebrae and sternum (both measured at the same axial level). If this ratio is above 3.25 , it is considered severe [1].

CT scanning has commonly been used for this purpose [1]. Recent reports in the literature, however, have recognized the problem of radiation exposure when using CT scanning for this purpose [2].

\section{Description}

At our institution, we have introduced the use of a fast MRI technique to measure the Haller index. This technique has proven reliable and easy to perform, and avoids the ionizing radiation of $\mathrm{CT}$ scanning.

MRI scanning at our institution for this purpose is performed on a $1.5 \mathrm{~T}$ HDxT platform (General Electric, Milwaukee, WI). An 8- or 12-channel body coil is used. A short localizer $(10 \mathrm{~s})$ is followed by a 2-D FIESTA (TE $1.7-1.8$, TR 3.7-3.8, 192 × 256, 1 NEX) fat-saturated sequence. Slice thickness is $5 \mathrm{~mm}$ with a $1 \mathrm{~mm}$ gap obtained through the lower chest. The time required for the FIESTA sequence is approximately $20 \mathrm{~s}$ for a 20 -slice acquisition. Breath-hold is not necessary within these scan parameters, although both inspiratory as well as expiratory acquisitions could be easily obtained with a minimum of added effort. Cardiac gating is not needed. Total scan time is under $5 \mathrm{~min}$. The specific MR sequence (FIESTA $=$ Fast Imaging Employing Steady-state Acquisition) was selected because it can produce high SNR images in a short acquisition time.

Measurement of the Haller index is performed in the standard manner using the electronic calipers on our PACS monitors. 


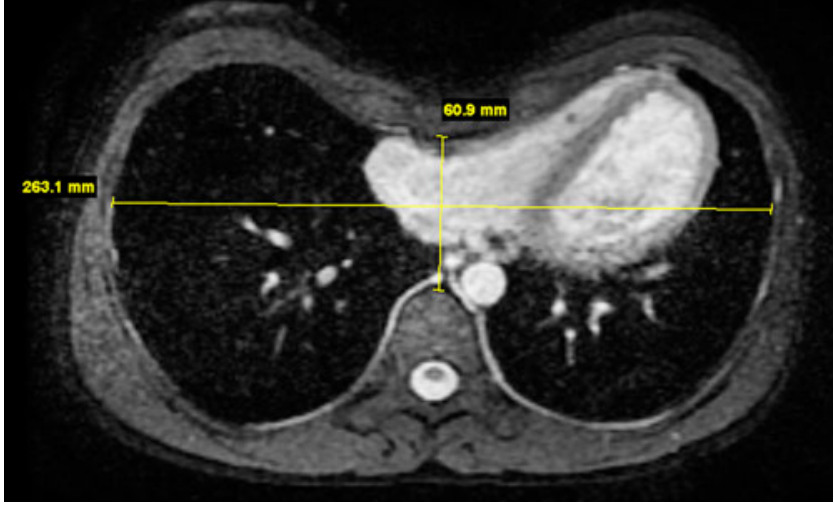

Fig. 1 Axial 2-D FIESTA of the chest demonstrates pectus excavatum with the Haller index measurement

\section{Discussion}

At our institution, MRI has replaced CT scanning for evaluating pectus excavatum and the measurement of the Haller index. A short localizer is followed by a single FIESTA (Phillips refers to this as balanced FFE; Siemens refers to this as trueFISP) acquisition. The entire MRI examination requires less than $5 \mathrm{~min}$ to perform, which compares favorably with CT scanning. While both MRI and $\mathrm{CT}$ produce diagnostic quality scans which can be easily interpreted, MRI requires no ionizing radiation. Since pectus excavatum repair is almost exclusively performed in older children and adolescents, no sedation is required.

At our site, our primary pectus excavatum surgeon has switched to ordering the MRI examination described above, and has reported that the results are reliable and depict well the relevant anatomy. To date, we have scanned approximately 50 patients using this MRI technique.

Other researchers have recently proposed using a twoview chest radiograph to replace CT scanning for the purpose of Haller index measurement. However, this still requires the use of ionizing radiation [3].

Though there is an association of bronchiectasis with pectus excavatum [4], the primary purpose of presurgical imaging in this condition is to assess the Haller index. In fact, single-slice CT scanning would also likely underestimate the degree of bronchiectasis. In patients with suspected bronchiectasis related to pectus excavatum, fullchest CT scanning may be the preferred presurgical image examination.

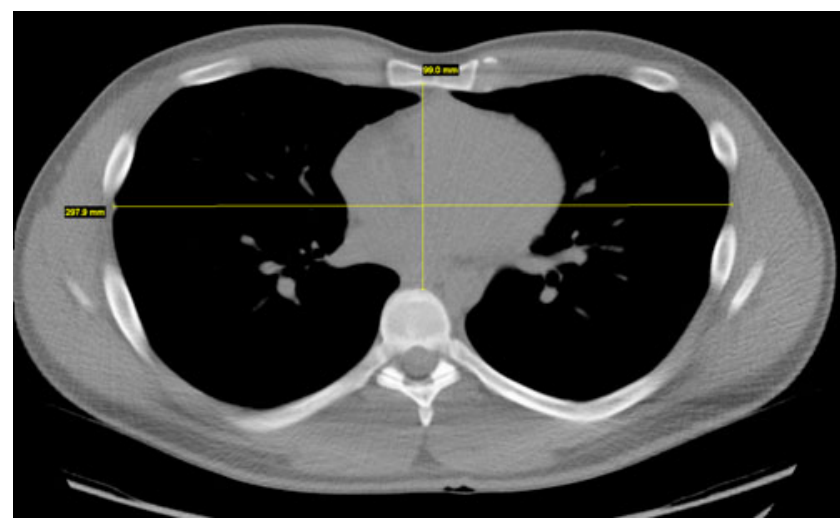

Fig. 2 Axial CT scan of the chest demonstrates pectus excavatum with the Haller index measurement

Future directions in the imaging evaluation of pectus excavatum include the routine acquisition of inspiratory and expiratory MRI sequences. Research has shown that this may provide more physiological information; in expiration, the deformity may worsen [5]. Furthermore, cine MR imaging has been shown to be capable of evaluating both chest morphology and chest wall kinetics, and may well add important diagnostic information [6] (Figs 1 and 2).

Open Access This article is distributed under the terms of the Creative Commons Attribution Noncommercial License which permits any noncommercial use, distribution, and reproduction in any medium, provided the original author(s) and source are credited.

\section{References}

1. Haller JA Jr, Kramer SS, Lietman SA et al (1987) Use of CT scans in selection of patients for pectus excavatum surgery: a preliminary report. J Pediatr Surg 22:904-906

2. Rattan AS, Laor T, Ryckman FC et al (2010) Pectus excavatum imaging: enough but not too much. Pediatr Radiol 40:168-172

3. Khanna G, Jaju A, Don S et al (2010) Comparison of Haller index values calculated with chest radiographs versus CT for pectus excavatum evaluation. Pediatr Radiol 40:1763-1767

4. Morshuis WJ, Mulder H, Wapperom G et al (1992) Pectus excavatum. A clinical study with long-term postoperative follow-up. Eur J Cardiothorac Surg 6:318-328, discussion 328-329

5. Raichura N, Entwisle J, Leverment J et al (2001) Breath-hold MRI in evaluating patients with pectus excavatum. Br J Radiol 74:701-708

6. Herrmann KA, Zech C, Strauss T et al (2006) Cine MRI of the thorax in patients with pectus excavatum. Radiologe 46:309-316, German 